\title{
Incidence, characteristics, and outcome of post-heart transplant malignancy
}

\author{
Jong-Chan Youn ${ }^{1}$, In-Cheol Kim², Jin-Jin Kim, Sang Hong Baek ${ }^{1}$, Jon Kobashigawa ${ }^{3}$ \\ ${ }^{1}$ Division of Cardiology, Department of Internal Medicine, Seoul St. Mary's Hospital, College of Medicine, The Catholic University of Korea, Seoul, Korea \\ ${ }^{2}$ Division of Cardiology, Department of Internal Medicine, Keimyung University Dongsan Medical Center, Daegu, Korea \\ ${ }^{3}$ Division of Cardiology, Department of Internal Medicine, Cedars-Sinai Medical Center, Beverly Hills, CA, USA
}

Background: Post-transplant malignancy (PTM) has been an important cause of long-term morbidity and mortality in heart transplant (HTx) recipients. However, the detailed characteristics of PTM are largely unknown. We aimed to describe the characteristics, outcomes, and predictors of de novo PTM in HTx recipients.

Methods: We analyzed 989 consecutively enrolled HTx recipients without pre-transplant history of malignancy between 1997 and 2013. Main outcomes included the incidence, characteristics, outcomes, and predictors of PTM.

Results: During a median follow-up period of 11.5 years, 206 patients (20.8\%) had de novo PTMs (241 cancer cases). PTM patients, compared with non-PTM patients, showed older age, longer time on immunosuppression, and higher proportion of male and white patients. Skin cancers were the most frequent types of malignancy $(60.6 \%)$ followed by prostate cancers $(9.5 \%)$, lung cancers $(7.1 \%)$, and breast cancers $(4.1 \%)$. While most of the cancers $(88.8 \%)$ were surgically resected at the initial presentation, about half of cancers $(47.3 \%)$ showed recurrence or disease progression. Both patients with skin cancer and non-skin cancer, compared to patients without PTM, revealed a significantly lower overall survival $(P<0.001)$. Multivariable analysis revealed older age $(P<0.001)$, white race $(P=0.001)$, and longer time on immunosuppression $(P<0.001)$ were independent predictors for PTM.

Conclusions: Older, white recipients with longer time on immunosuppression were independent risk factors for PTM, which was associated with increased mortality. Enhanced cancer screening and individualized immunosuppression in these high-risk patients should be studied to improve the outcomes.

Corresponding author: Jong-Chan Youn

E-mail: jong.chan.youn@gmail.com

(C) The Korean Society for Transplantation

This is an Open Access article distributed under the terms of the Creative Commons Attribution Non-Commercial License (http://creativecommons.org/licenses/by-nc/4.0/) which permits unrestricted non-commercial use, distribution, and reproduction in any medium, provided the original work is properly cited. 